\title{
Transient Multimedia Model for Investigating the Influence of Indoor Human Activities on Exposure to SVOCs
}

Jacob Kvasnicka ${ }^{1}$, Elaine Cohen $\mathrm{Hubal}^{2}$, John Ladan ${ }^{3}$, Xianming Zhang ${ }^{1}$, Miriam L. Diamond*1,4

${ }^{1}$ Department of Earth Sciences, University of Toronto, Toronto, Ontario, Canada

${ }^{2}$ Center for Public Health and Environmental Assessment, Environmental Protection Agency, Durham, North Carolina, U.S.A.

${ }^{3}$ Department of Physics, University of Toronto, Toronto, Ontario, Canada

${ }^{4}$ Dalla Lana School of Public Health, University of Toronto, Toronto, Ontario, Canada

* Corresponding Author:

Miriam L. Diamond

Department of Earth Sciences

22 Ursula Franklin Street

University of Toronto

Toronto, Ontario, Canada M5S 3B1

1 (416) 978-1586

miriam.diamond@utoronto.ca 


\section{Contents}

Modeling Particle-Mediated SVOC Mass Transfer .............................................................. 3

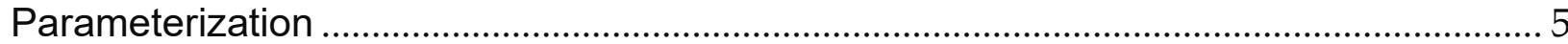

Table S1. Environmental parameterization for a single-room residence in North America. ..... 5

Table S2. Physical-chemical parameterization for three phthalates: DEP, BBzP, and DEHP... 6

Table S3. Particle parameterization for three size-ranges: $\mathrm{PM}_{2.5}, \mathrm{PM}_{2.5-10}$, and $\mathrm{PM}_{10-150} \ldots \ldots \ldots . .7$

Table S4. Human parameterization for an archetypal adult and toddler. ................................... 8

Table S5. Occupant activity schedules. ........................................................................... 9

Table S6. Equations for deriving fugacity capacities ........................................................ 10

Table S7. Equations for deriving mass-transfer coefficients .................................................. 13

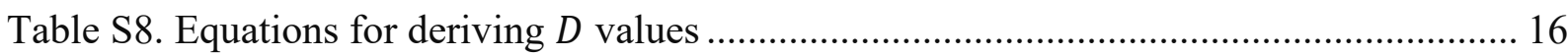

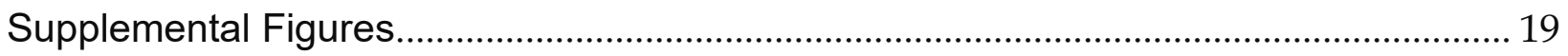

Figure S1. Dynamics of internal and external phthalate exposure for the archetypal adult male occupant.

Figure S2. Dynamics of internal and external phthalate exposure for the archetypal toddler occupant.

Figure S3. Dynamics of phthalates in other surfaces (top graph) and indoor air (bottom graph) for the archetypal male adult occupant's activity exposure simulation..................................... 21

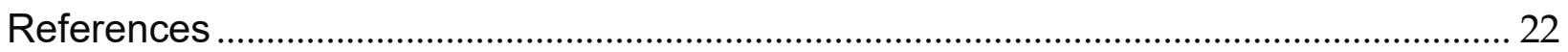




\section{Modeling Particle-Mediated SVOC Mass Transfer}

The following is a general mathematical expression, in fugacity notation, ${ }^{1}$ for the multimedia dynamics of a neutral organic compound in any two bulk compartments $i$ and $j$ :

$$
\frac{d m_{i}}{d t}=\frac{d\left(V_{i} Z_{i} f_{i}\right)}{d t}=E_{i}+\sum_{\substack{j=1 \\ j \neq i}}^{9} D_{j \rightarrow i} f_{j}-\left(D_{i, \text { rem }}+\sum_{\substack{j=1 \\ j \neq i}}^{9} D_{i \rightarrow j}\right) f_{i}
$$

where $m_{i}$ [mol] and $f_{i}[\mathrm{~Pa}]$ are the mass and fugacity, respectively, of the compound in compartment $i ; V_{i}$ is the volume of the bulk compartment $\left[\mathrm{m}^{3}\right] ; Z_{i}$ is the fugacity capacity $\left[\mathrm{mol} \cdot \mathrm{m}^{-3} \cdot \mathrm{Pa}^{-1}\right]$, which quantifies the compartment's storage capacity for the compound; $E_{i}$ is the emission rate $\left[\mathrm{mol} \cdot \mathrm{h}^{-1}\right] ; D_{i \rightarrow j}, D_{j \rightarrow i}$, and $D_{i, r e m}$ are " $D$ values" $\left[\mathrm{mol} \cdot \mathrm{h}^{-1} \cdot \mathrm{Pa}^{-1}\right]$ quantifying rates of mass transfer $(\rightarrow)$ from compartment $i$ to $j$ and vice versa or removal from compartment $i$ (rem).

For compartments in which $V_{i}$ and $Z_{i}$ do not vary with time, Equation $\mathbf{S} 1$ can be simplified to the following expression with $f_{i}$ as the state variable:

$$
\frac{d f_{i}}{d t}=\frac{E_{i}}{V_{i} Z_{i}}+\sum_{\substack{j=1 \\ j \neq i}}^{9} \frac{D_{j \rightarrow i}}{V_{i} Z_{i}} f_{j}-\left(\frac{D_{i, r e m}}{V_{i} Z_{i}}+\sum_{\substack{j=1 \\ j \neq i}}^{9} \frac{D_{i \rightarrow j}}{V_{i} Z_{i}}\right) f_{i}
$$

In ABICAM, this equation is applicable to the human compartments (hands, skin, internal body), because these compartments do not accumulate or lose time-varying particle mass as is the case with other compartments.

In contrast, ABICAM's six environmental compartments (indoor air, polyurethane foam, hard floor, carpet, up-facing surfaces, other surfaces) can accumulate or lose time-varying particle mass. These time dependencies are represented by the following two equations:

$$
\begin{gathered}
V_{i}(t)=V_{i, p u r e}+\sum_{s} V_{p, s, i}(t) \\
Z_{i}(t)=Z_{i, p u r e}+\sum_{s} Z_{p, s, i} \cdot \varphi_{p, s, i}(t)
\end{gathered}
$$

where $V_{i, p u r e}$ and $Z_{i, p u r e}$ are the volume and fugacity capacity, respectively, of the pure phase within compartment $i ; V_{p, s, i}(t)$ and $Z_{p, s, i}$ are the volume and fugacity capacity, respectively, of particles of size range $s$ in the bulk compartment $i ; \varphi_{p, s, i}(t)=\left(\frac{V_{p, s, i}(t)}{V_{i}(t)}\right)$ is the time-dependent particle volume fraction $\left[\mathrm{m}^{3}{ }_{\mathrm{s}} / \mathrm{m}^{3} \mathrm{i}\right]$. 
To ensure mass conservation, the product rule must be used with Equation $\mathbf{S 1}$ to derive Equation 2 in the main text with its two "correction terms," $V_{i}^{\prime} / V_{i}$ and $Z_{i}^{\prime} / Z_{i}$. These terms are responsible for changes in chemical fugacity that result from changes in $V_{i}$ and $Z_{i}$, respectively. ${ }^{2}$ To understand these correction terms, consider a compartment in chemical equilibrium and at steady state, $\frac{d m_{i}}{d t}=V_{i} Z_{i} \frac{d f_{i}}{d t}+f_{i} Z_{i} \frac{d V_{i}}{d t}+f_{i} V_{i} \frac{d Z_{i}}{d t}=0$. A change in bulk volume or fugacity capacity would change the fugacity through the relation

$$
\frac{d f_{i}}{d t}=-\left(\frac{V_{i}{ }^{\prime}}{V_{i}}+\frac{Z_{i}{ }^{\prime}}{Z_{i}}\right) f_{i}
$$

without a change in mass. This change in fugacity would push the compartment out of equilibrium, because fugacity is the equilibrium criterion. For example, if $a$ denotes the bulk indoor air compartment, it can be shown from this simplified expression how a rapid increase in the mass of particles in indoor air (e.g., from cooking) can contribute to elevated masses of the lower-volatility phthalates, butyl benzyl phthalate (BBzP) and di-2-ethylhexyl phthalate (DEHP). If particles are emitted to indoor air, the values of $Z_{a}$ for these compounds will increase according to Table S5 Equation S9. Assuming instantaneous equilibrium, these phthalates would then be removed from the gas phase as they partition to particles. Consequently, $f_{a}$ would decrease according to Equation S5. This decrease in $f_{a}$ would increase the fugacity gradient or driving force between the air compartment and compartment $j$. The net effect would be an increase in the rate of mass transfer from compartment $j$ to the air compartment, because the rate of diffusion between them, $N_{V, a \leftrightarrow j}\left[\mathrm{~mol} \cdot \mathrm{h}^{-1}\right]$, is directly proportional to the fugacity gradient $\Delta f_{a, j}[\mathrm{~Pa}]: N_{V, a \leftrightarrow j}=D_{V, a \leftrightarrow j} \cdot \Delta f_{a, j}$.

This phenomenon of enhanced diffusive mass transfer to indoor air is illustrated in the time-series results in Figure S3. As shown, elevated concentrations of total suspended particles, primarily from the adult's cooking activities, resulted in a net reduction of approximately 175 nmol of DEHP in surfaces. These losses were driven by enhanced diffusive transfer of DEHP to indoor air, which also occurred for the other environmental compartments (polyurethane foam mattress, hard floor, carpet, and upward-oriented surfaces), offset by particle deposition. The net effects were transient increases in the amount of BBzP and DEHP in indoor air. 


\section{Parameterization}

Table S1. Environmental parameterization for a single-room residence in North America.

\begin{tabular}{|c|c|c|c|}
\hline Parameter & Units & Value & Description \\
\hline$\overline{A_{\text {room }}}$ & $\mathrm{m}^{2}$ & 25 & Cross-sectional surface area of the room (indoor air compartment) \\
\hline$h_{\text {room }}$ & $\mathrm{m}$ & 3 & Height of the room (indoor air compartment) \\
\hline$A_{P U F}$ & $\mathrm{~m}^{2}$ & 1.84 & Surface area of PUFa,b \\
\hline$h_{P U F}$ & $\mathrm{~m}$ & 0.08 & Thickness of PUF ${ }^{\mathrm{a}, \mathrm{b}}$ \\
\hline$A_{\text {floor }}$ & $\mathrm{m}^{2}$ & 15 & Surface area of hard floor \\
\hline$h_{\text {floor }}$ & $\mathrm{m}$ & $5 \cdot 10^{-4}$ & Thickness of hard floor \\
\hline$A_{\text {carpet }}$ & $\mathrm{m}^{2}$ & 10 & Surface area of carpet \\
\hline$h_{\text {carpet }}$ & $\mathrm{m}$ & 0.01 & Thickness of carpet (fibers only) \\
\hline$A_{\text {surf_up }}$ & $\mathrm{m}^{2}$ & 60 & Total surface area of up-facing surfaces \\
\hline$A_{\text {surf_other }}$ & $\mathrm{m}^{2}$ & 140 & Total area of vertical- and down-facing surfaces \\
\hline$h_{\text {surf }}$ & $\mathrm{m}$ & $1 \cdot 10^{-7}$ & Thickness of surface film \\
\hline$\rho_{\text {film }}$ & $\mathrm{kg} \cdot \mathrm{m}^{-3}$ & 1200 & Density of surface film \\
\hline$\varphi_{P U F, A}$ & $\mathrm{~m}^{3} \cdot \mathrm{m}^{-3}$ & 0.98 & Volume fraction of pore air in $\mathrm{PUF}^{\mathrm{b}}$ \\
\hline$\varphi_{\text {carpet }, A}$ & $\mathrm{~m}^{3} \cdot \mathrm{m}^{-3}$ & 0.89 & Volume fraction of pore air in carpet \\
\hline$c$ & unitless & $9 \cdot 10^{-3}$ & Correction factor for deriving effective diffusivity in porous media ${ }^{\mathrm{c}}$ \\
\hline$F O C_{P U F}$ & $\mathrm{~m}^{3} \cdot \mathrm{m}^{-3}$ & 0.65 & Fraction of organic carbon in PUF ${ }^{b}$ \\
\hline FOC floor & $\mathrm{m}^{3} \cdot \mathrm{m}^{-3}$ & 0.37 & Fraction of organic carbon in hard floor \\
\hline FOC $C_{\text {carpet }}$ & $\mathrm{m}^{3} \cdot \mathrm{m}^{-3}$ & 0.64 & Fraction of organic carbon in carpet \\
\hline FOC film $_{1}$ & $\mathrm{~m}^{3} \cdot \mathrm{m}^{-3}$ & 0.2 & Fraction of organic carbon in surface film \\
\hline$\delta_{b l}$ & $\mathrm{~m}$ & $5 \cdot 10^{-3}$ & Thickness of the air-side boundary layer \\
\hline$A E R$ & $h^{-1}$ & 0.45 & Air exchange rate ${ }^{\mathrm{d}}$ \\
\hline
\end{tabular}

Note: Parameter values were adopted from Zhang et al. $(2014)^{3}$ unless indicated by a footnote.

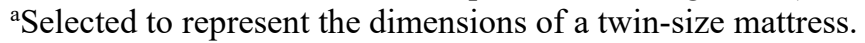

bPUF, polyurethane foam.

${ }^{\mathrm{c}}$ Adopted from Zhang et al. $2011^{4}$ to account for reduced diffusion in PUF and carpet (assuming similar masstransfer properties) due to tortuosity.

${ }^{\mathrm{d}}$ Recommended by U.S. Environmental Protection Agency $(2011)^{5}$ as a central estimate for all single-family homes, including mobile homes and multifamily units in the United States. 
Table S2. Physical-chemical parameterization for three phthalates: DEP, BBzP, and DEHP.

\begin{tabular}{|c|c|c|c|c|c|}
\hline Parameter & Units & DEP & $\mathrm{BBzP}$ & DEHP & Description \\
\hline$\overline{M W}$ & $\mathrm{~g} \cdot \mathrm{mol}^{-1}$ & 222.24 & 312.37 & 390.57 & Molecular weight \\
\hline $\log _{10} K_{A W}$ & unitless & -5.0 & -5.9 & -4.1 & $\begin{array}{l}\text { Base- } 10 \text { logarithm of air-water } \\
\text { partition coefficient at } 25^{\circ} \mathrm{C}\end{array}$ \\
\hline $\log _{10} K_{O W}$ & unitless & 2.4 & 4.7 & 7.6 & $\begin{array}{l}\text { Base- } 10 \text { logarithm of octanol- } \\
\text { water partition coefficient at } 25^{\circ} \mathrm{C}\end{array}$ \\
\hline $\log _{10} K_{O A}$ & unitless & 7.4 & 10.6 & 11.7 & $\begin{array}{l}\text { Base-10 logarithm of octanol-air } \\
\text { partition coefficient at } 25^{\circ} \mathrm{C}\end{array}$ \\
\hline$\Delta U_{A W}$ & $\mathrm{~kJ} \cdot \mathrm{mol}^{-1}$ & 54 & 82 & 92 & $\begin{array}{l}\text { Internal energy of phase change } \\
\text { (air-water) }\end{array}$ \\
\hline$\Delta U_{O W}$ & $\mathrm{~kJ} \cdot \mathrm{mol}^{-1}$ & -20 & -20 & -20 & $\begin{array}{l}\text { Internal energy of phase change } \\
\text { (octanol-water) }\end{array}$ \\
\hline$\Delta U_{O A}$ & $\mathrm{~kJ} \cdot \mathrm{mol}^{-1}$ & -74 & -102 & -112 & $\begin{array}{l}\text { Internal energy of phase change } \\
\text { (octanol-air) }\end{array}$ \\
\hline$\tau_{\text {deg,air }}$ & $\mathrm{h}$ & 510 & 160 & 80 & $\begin{array}{l}\text { Degradation half-life in indoor air } \\
\text { for reaction with hydroxyl radical }\end{array}$ \\
\hline$\tau_{\text {deg,body }}$ & $\mathrm{h}$ & 4.3 & 4.3 & 4.3 & Human metabolic half-life \\
\hline$k_{\text {rem,wash }}$ & $\mathrm{s}^{-1}$ & 0.02 & 0.01 & 0.01 & $\begin{array}{l}\text { First-order removal rate } \\
\text { coefficient for washing skin }\end{array}$ \\
\hline
\end{tabular}

Note: Parameter values were adopted from Zhang et al. (2014), ${ }^{3}$ with additional detail regarding the estimation of internal energies of phase change in the user manual for their model.

${ }^{a}$ A common half-life was chosen for all three compounds, given uncertainties in elimination kinetics and similar half-lives $(<24 \mathrm{~h})$ observed across a diverse suite of phthalates. ${ }^{6}$ This estimate is based on the mean terminal elimination half-life for DEHP across human participants in a study by Kessler et al. (2012). ${ }^{7}$

${ }^{b}$ First-order removal rate coefficients for handwashing were derived from a study by Gong et al. (2014). ${ }^{8}$ These coefficients were also applied to the showering activity, assuming similar removal rates. 
Table S3. Particle parameterization for three size-ranges: $\mathrm{PM}_{2.5}, \mathrm{PM}_{2.5-10}$, and $\mathrm{PM}_{10-150}$.

\begin{tabular}{|c|c|c|c|c|c|}
\hline Parameter & Units & $\mathrm{PM}_{2.5}$ & $\mathrm{PM}_{2.5-10}$ & $\mathrm{PM}_{10-150}$ & Description \\
\hline$\rho_{p, s}$ & $\mathrm{~kg} \cdot \mathrm{m}^{-3}$ & 1000 & 1000 & 1000 & Density \\
\hline$F O C_{p, s}$ & $\mathrm{~m}^{3} \cdot \mathrm{m}^{-3}$ & 0.65 & 0.28 & 0.06 & Fraction of organic carbon \\
\hline$k_{\text {dep }, \text { up }}$ & $\mathrm{m} \cdot \mathrm{h}^{-1}$ & 0.1 & 10 & 608 & Deposition velocity to up-facing surfaces ${ }^{a}$ \\
\hline$k_{\text {dep,vert }}$ & $\mathrm{m} \cdot \mathrm{h}^{-1}$ & 0.02 & 2 & 122 & Deposition velocity to vertical surfaces ${ }^{a}$ \\
\hline$k_{\text {floor } \rightarrow \text { air }}$ & $\mathrm{h}^{-1}$ & $3 \cdot 10^{-3}$ & $2 \cdot 10^{-2}$ & $3 \cdot 10^{-2}$ & Resuspension rate for floor ${ }^{b}$ \\
\hline$k_{\text {carpet } \rightarrow \text { air }}$ & $\mathrm{h}^{-1}$ & $3 \cdot 10^{-3}$ & $2 \cdot 10^{-2}$ & $3 \cdot 10^{-2}$ & Resuspension rate for carpet ${ }^{b}$ \\
\hline$k_{P U F \rightarrow a i r}$ & $h^{-1}$ & $6 \cdot 10^{-3}$ & $4 \cdot 10^{-2}$ & $6 \cdot 10^{-2}$ & Resuspension rate for $\mathrm{PUF}^{\mathrm{c}, \mathrm{d}}$ \\
\hline$k_{\text {rem }, P U F}$ & $h^{-1}$ & $2 \cdot 10^{-5}$ & $2 \cdot 10^{-5}$ & $2 \cdot 10^{-5}$ & Removal rate for PUF ${ }^{\mathrm{e}}$ \\
\hline$k_{\text {rem,floor }}$ & $h^{-1}$ & $1 \cdot 10^{-3}$ & $1 \cdot 10^{-3}$ & $1 \cdot 10^{-3}$ & Removal rate for floor ${ }^{\mathrm{e}}$ \\
\hline$k_{\text {rem,carpet }}$ & $h^{-1}$ & $2 \cdot 10^{-4}$ & $2 \cdot 10^{-4}$ & $2 \cdot 10^{-4}$ & Removal rate for carpet ${ }^{\mathrm{e}}$ \\
\hline$k_{\text {rem,surf_up }}$ & $h^{-1}$ & $2 \cdot 10^{-4}$ & $2 \cdot 10^{-4}$ & $2 \cdot 10^{-4}$ & Removal rate for up-facing surfaces ${ }^{e}$ \\
\hline$k_{\text {rem,surf_other }}$ & $\mathrm{h}^{-1}$ & $2 \cdot 10^{-5}$ & $2 \cdot 10^{-5}$ & $2 \cdot 10^{-5}$ & Removal rate for other surfaces ${ }^{e}$ \\
\hline$C_{p, s, \text { outdoor }}$ & $\mu \mathrm{g} \cdot \mathrm{m}^{-3}$ & 7.3 & 6.9 & 16 & Particle concentration in outdoor air \\
\hline$P E_{p, s, \text { outdoor }}$ & unitless & 0.82 & 0.65 & 0.10 & $\begin{array}{l}\text { Penetration efficiency of outdoor particles } \\
\text { through the building envelope }\end{array}$ \\
\hline$s_{s, \text { air }, \text { cooking }}$ & $\mu g \cdot h^{-1}$ & 3125 & 833 & 0 & $\begin{array}{l}\text { Source term or emission rate associated } \\
\text { with cooking }\end{array}$ \\
\hline
\end{tabular}

Note: Parameter values were adopted from Shin et al. (2013) $)^{9}$ unless indicated by a footnote. Some of these parameters are activity-dependent and thus time-dependent as explained in the main text.

${ }^{a}$ Deposition rate coefficients were adopted from Zhang et al. (2014). ${ }^{3}$ Coefficients for downward-oriented surfaces were assumed to be negligible.

${ }^{b}$ Resuspension rate coefficients for hard floor and carpet were adopted from Bramwell et al. (2016). ${ }^{10}$ These coefficients were also applied to the toddler, due to a lack of available data for this archetype. Body mass has been observed to have a minimal influence on resuspension from mattresses. ${ }^{11}$

'PUF, polyurethane foam.

${ }^{\mathrm{d}}$ Resuspension rate coefficients for PUF were applied to the toddler's bouncing activity. These coefficients were derived from $k_{\text {carpet } \rightarrow \text { air }}$ multiplied by a factor of two to account for heavy and fast movement based on findings from Qian et al. (2008). ${ }^{12}$

${ }^{e}$ Removal rate coefficients were only applied to derive a steady-state solution of the initial value problem, because they implicitly assume a frequency of dust removal (e.g., from cleaning), which was not evaluated as an activity in this case study. 
Table S4. Human parameterization for an archetypal adult and toddler.

\begin{tabular}{|c|c|c|c|c|}
\hline Parameter & Units & Adult & Toddler $^{1}$ & Description \\
\hline$T_{\text {skin }}$ & ${ }^{\circ} \mathrm{C}$ & 32 & 32 & Temperature of skin (including hands) \\
\hline$T_{\text {body }}$ & ${ }^{\circ} \mathrm{C}$ & 37 & 37 & Temperature of internal body \\
\hline$B W$ & $\mathrm{~kg}$ & 84 & 14 & Body weight ${ }^{\mathrm{a}}$ \\
\hline$\varphi_{\text {bulk,body }}$ & $\mathrm{kg} \cdot \mathrm{m}^{-3}$ & 1000 & 1000 & Bulk density of internal body \\
\hline$f_{\text {lipid,body }}$ & $\mathrm{kg} \cdot \mathrm{kg}^{-1}$ & 0.2 & 0.2 & Mass fraction of lipids in body \\
\hline$\varphi_{\text {lipid,body }}$ & $\mathrm{kg} \cdot \mathrm{m}^{-3}$ & 900 & 900 & Density of lipids in body \\
\hline$f_{\text {water,body }}$ & $\mathrm{kg} \cdot \mathrm{kg}^{-1}$ & 0.6 & 0.6 & Mass fraction of water in body \\
\hline$\varphi_{\text {water, body }}$ & $\mathrm{kg} \cdot \mathrm{m}^{-3}$ & 1000 & 1000 & Density of water in body \\
\hline$f_{N L O M, b o d y}$ & $\mathrm{~kg} \cdot \mathrm{kg}^{-1}$ & 0.2 & 0.2 & $\begin{array}{l}\text { Mass fraction of non-lipid organic matter } \\
\text { in body }\end{array}$ \\
\hline$\varphi_{\text {NLOM,body }}$ & $\mathrm{kg} \cdot \mathrm{m}^{-3}$ & 1000 & 1000 & Density of non-lipid organic matter in body \\
\hline$A_{\text {skin }}$ & $\mathrm{m}^{2}$ & 2.08 & 0.62 & Surface area of skin (including hands) ${ }^{\mathrm{a}}$ \\
\hline$A_{\text {hands }}$ & $\mathrm{m}^{2}$ & 0.104 & 0.032 & Surface area of hands ${ }^{\mathrm{a}}$ \\
\hline$h_{\text {lipid }}$ & $\mu \mathrm{m}$ & 0.6 & 0.6 & Thickness of skin-surface lipids \\
\hline$G_{\text {resp }, 1}$ & $\mathrm{~m}^{3} \cdot \mathrm{min}^{-1} \cdot \mathrm{kg}^{-1}$ & $6.6 \cdot 10^{-5}$ & $2.5 \cdot 10^{-4}$ & $\begin{array}{l}\text { Respiration rate for sedentary/passive } \\
\text { activities (METS } \leq 1.5)^{\mathrm{b}}\end{array}$ \\
\hline$G_{\text {resp }, 2}$ & $\mathrm{~m}^{3} \cdot \mathrm{min}^{-1} \cdot \mathrm{kg}^{-1}$ & $1.6 \cdot 10^{-4}$ & $6.3 \cdot 10^{-4}$ & $\begin{array}{l}\text { Respiration rate for light-intensity } \\
\text { activities (METS } 1.5-3.0)^{\mathrm{b}}\end{array}$ \\
\hline$G_{\text {resp }, 3}$ & $\mathrm{~m}^{3} \cdot \mathrm{min}^{-1} \cdot \mathrm{kg}^{-1}$ & - & $2.2 \cdot 10^{-3}$ & $\begin{array}{l}\text { Respiration rate for high-intensity activities } \\
(\text { METS }>6.0)^{b}\end{array}$ \\
\hline$G_{\text {urine }}$ & $m^{3 \cdot} d^{-1}$ & $1.1 \cdot 10^{-3}$ & $5.5 \cdot 10^{-4}$ & Urination rate $\mathrm{c}^{\mathrm{c}}$ \\
\hline$k_{r e m, s c}$ & $\mathrm{~d}^{-1}$ & $6.7 \cdot 10^{-2}$ & $6.7 \cdot 10^{-2}$ & Frequency of skin-cell regeneration \\
\hline$T E$ & unitless & - & 0.48 & $\begin{array}{l}\text { Chemical mass transfer efficiency of hand- } \\
\text { mouth contact }^{\mathrm{d}}\end{array}$ \\
\hline$n_{c}$ & $h^{-1}$ & - & 13 & Frequency of hand-mouth contact ${ }^{\mathrm{a}}$ \\
\hline$A_{\text {hand_mouth }}$ & $\mathrm{m}^{2}$ & - & $1.9 \cdot 10^{-3}$ & $\begin{array}{l}\text { Surface area of hand that is mouthed } \\
\text { during hand-mouth contact }\end{array}$ \\
\hline$\varphi_{P U F, A, \text { bounce }}$ & $\mathrm{m}^{3} \cdot \mathrm{m}^{-3}$ & - & 0.1 & $\begin{array}{l}\text { Fraction of PUF air that is exchanged with } \\
\text { room air during one bounce }\end{array}$ \\
\hline$n_{\text {bounce }}$ & $\min ^{-1}$ & - & 60 & $\begin{array}{l}\text { Frequency of compression of PUF during } \\
\text { bouncing }\end{array}$ \\
\hline
\end{tabular}

Note: Parameter values were adopted from Zhang et al. $(2014)^{3}$ for an archetypal male adult, unless indicated by a footnote. For some parameters, the adult's values were also used for the toddler in the absence of data.

${ }^{a}$ Central estimate for the toddler adopted from U.S Environmental Protection Agency (EPA) (2011). ${ }^{5}$

bMETS, metabolic equivalents. $G_{r e s p, 1}$ was applied to the sleeping activity and $G_{r e s p, 3}$ was applied to the bouncingon-mattress activity for the toddler. $G_{\text {resp }, 2}$ was applied to all other activities. These parameter values are central estimates adopted from U.S. EPA (2011). ${ }^{5}$

${ }^{\mathrm{c}}$ Based on nationally-representative data on water consumption from fluids in the United States, excluding water in foods. ${ }^{13}$

${ }^{\mathrm{d}}$ Recommended by U.S. EPA (2012) $)^{14}$ as a central estimate.

ePUF., polyurethane foam. Assuming an average of one bounce per second for a short (10 minute) duration, with $10 \%$ compression per bounce. 
Table S5. Occupant activity schedules.

\begin{tabular}{|c|c|c|c|}
\hline Adult activity & Start-time & Toddler activity & Start-time \\
\hline Walk on hard floor & 07:00:00 & Walk on hard floor & 07:00:00 \\
\hline Shower & 07:45:00 & Hand-mouth contacts & $07: 52: 00$ \\
\hline Wash hands & $07: 58: 30$ & Wash hands & 09:52:00 \\
\hline Cook & 07:59:05 & Walk on hard floor & $09: 52: 35$ \\
\hline Idle & 08:39:05 & Sleep & $12: 30: 35$ \\
\hline Wash hands & $09: 42: 30$ & Walk on carpet & $14: 31: 00$ \\
\hline Walk on hard floor & 09:43:05 & Hand-mouth contacts & $16: 51: 00$ \\
\hline Wash hands & $13: 08: 30$ & Wash hands & 17:00:00 \\
\hline Idle & 13:09:05 & Sleep & $17: 00: 35$ \\
\hline Wash hands & $13: 43: 30$ & Bounce on mattress & $18: 49: 00$ \\
\hline Cook & $13: 44: 05$ & Hand-mouth contacts & 18:59:00 \\
\hline Idle & $14: 04: 05$ & Wash hands & $23: 00: 00$ \\
\hline Wash hands & $15: 03: 30$ & Sleep & $23: 00: 35$ \\
\hline Walk on carpet & $15: 04: 05$ & - & - \\
\hline Idle & $17: 21: 30$ & - & - \\
\hline Wash hands & $22: 59: 00$ & - & - \\
\hline Sleep & $22: 59: 35$ & - & - \\
\hline
\end{tabular}

Note: Activities for each occupant are characterized by a name and start-time (hour:minute:second). Parameter values are based on central tendencies of survey data. ${ }^{5}$ Corresponding mass-transfer and removal processes are summarized in Table 1 of the main text. 
Table S6. Equations for deriving fugacity capacities $\left[\mathrm{Z}, \mathrm{mol} \cdot \mathrm{m}^{-3} \cdot \mathrm{Pa}^{-1}\right]$.

\begin{tabular}{|c|c|c|c|}
\hline Phase & Equation & & Parameter Description \\
\hline Gas & $Z_{G}=\frac{1}{R \cdot T}$ & (S6) & $\begin{array}{l}\text { - } R: \text { Ideal Gas Law Constant } \\
{\left[8.314 \mathrm{~Pa} \cdot \mathrm{m}^{-3} \cdot \mathrm{mol}^{-1} \cdot \mathrm{K}^{-1}\right]} \\
\text { - } \quad T: \text { ambient temperature } \\
(298.15 \mathrm{~K})\end{array}$ \\
\hline $\begin{array}{l}\text { Particle }(s=0-2.5 \mu \mathrm{m}, 2.5-10 \\
\mu \mathrm{m}, \text { or } 10-150 \mu \mathrm{m} \text { size range })\end{array}$ & $\begin{array}{c}Z_{p, s}=K_{p, s} \cdot \rho_{p, s} \cdot 10^{-9} \cdot Z_{G} \\
\text { where } \log _{10}\left(K_{p, s}\right)=\log _{10}\left(K_{O A}\right)+\log _{10}\left(\frac{F O C_{p, s}}{0.74}\right)-11.91\end{array}$ & $\begin{array}{l}\text { (S7) } \\
(\mathrm{S} 8)\end{array}$ & $\begin{array}{l}K_{p, s}: \text { particle-gas partition } \\
\text { coefficient adopted from } \\
\text { Harner \& Bidleman }(1998)^{15} \\
\rho_{p, s}: \text { density }\left[\mathrm{kg} \cdot \mathrm{m}^{-3}\right] \text { of } \\
\text { particles in size range } s \\
\text { FOC } C_{p, s}: \text { fraction of organic } \\
\text { carbon of particles in size } \\
\text { range } s\end{array}$ \\
\hline Air & $Z_{A}=Z_{G}+\sum_{s=1}^{3} Z_{p, s} \cdot \varphi_{p, s, A}$ & (S9) & $\begin{array}{l}\text { - } \varphi_{p, s, A}: \text { volume fraction of } \\
\text { particles of size range } s \text { in bulk } \\
\text { indoor air }\end{array}$ \\
\hline Water & $Z_{W}=\frac{Z_{G}}{K_{A W}}$ & (S10) & $\begin{array}{ll}-K_{A W}: \text { air-water partition } \\
\text { coefficient [unitless] }\end{array}$ \\
\hline Lipid & $Z_{\text {lipid }}=Z_{W} \cdot K_{\text {OW }}$ & (S11) & $\begin{array}{l}\text { - } K_{O W} \text { : octanol-water partition } \\
\text { coefficient [unitless] }\end{array}$ \\
\hline Non-lipid organic matter & $Z_{N L O M}=0.035 \cdot Z_{\text {lipid }}$ & $(\mathrm{S} 12)$ & $\begin{array}{l}-0.035 \text { is a proportionality } \\
\text { constant }\end{array}$ \\
\hline PUF & $\begin{array}{c}Z_{P U F}=\varphi_{P U F, A} \cdot Z_{G}+\varphi_{P U F, \text { pure }} \cdot Z_{P U F, \text { pure }}+\sum_{s=1}^{3} \varphi_{p, s, P U F} \cdot Z_{p, s} \\
\text { where } Z_{P U F, \text { pure }}=K_{P U F, A} \cdot Z_{G} \\
\text { and } K_{P U F, A}=K_{O A} \cdot F O C_{P U F}\end{array}$ & $\begin{array}{l}(\mathrm{S} 13) \\
(\mathrm{S} 14) \\
(\mathrm{S} 15)\end{array}$ & $\begin{array}{ll}\text { - } & \varphi_{P U F, A}: \text { volume fraction of } \\
& \text { pore air in bulk PUF } \\
\text { - } & \varphi_{P U F, \text { pure }}: \text { volume fraction of } \\
& \text { pure PUF material in bulk PUF } \\
\text { - } & Z_{P U F, p u r e}: \text { fugacity capacity } \\
& {\left[\mathrm{mol}^{-3} \cdot \mathrm{m}^{-3} \cdot \mathrm{Pa}^{-1}\right] \text { of pure PUF }} \\
& \text { material } \\
\text { - } & K_{P U F, A}: \text { PUF-air partition } \\
& \text { coefficient [unitless] } \\
\text { - } & F O C_{P U F}: \text { fraction of organic } \\
& \text { carbon in PUF } \\
\end{array}$ \\
\hline
\end{tabular}

(Continued on next page) 
Table S6 Continued

\begin{tabular}{|c|c|c|c|}
\hline Phase & Equation & & Parameter Description \\
\hline Hard floor & $\begin{array}{c}Z_{\text {floor }}=\varphi_{\text {floor }, \text { pure }} \cdot Z_{\text {floor }, \text { pure }}+\sum_{s=1}^{3} \varphi_{p, s, \text { floor }} \cdot Z_{p, s} \\
\text { where } Z_{\text {floor,pure }}=K_{\text {floor }, A} \cdot Z_{G} \\
\text { and } K_{\text {floor }, A}=K_{O A} \cdot F O C_{f l o o r}\end{array}$ & $\begin{array}{l}(\mathrm{S} 16) \\
(\mathrm{S} 17) \\
(\mathrm{S} 18)\end{array}$ & $\begin{array}{l}\text { - } \varphi_{\text {floor,pure }}: \text { volume fraction of } \\
\text { pure hard floor in bulk hard } \\
\text { floor } \\
\text { - } Z_{\text {floor,pure }}: \text { fugacity capacity } \\
{\left[\mathrm{mol} \cdot \mathrm{m}^{-3} \cdot \mathrm{Pa}^{-1}\right] \text { of pure hard }} \\
\text { floor } \\
\text { - } K_{\text {floor, },}: \text { floor-air partition } \\
\text { coefficient [unitless] } \\
\text { - } F O C_{\text {floor }}: \text { fraction of organic } \\
\text { carbon in hard floor }\end{array}$ \\
\hline Carpet & $\begin{array}{c}Z_{\text {carpet }}=\varphi_{\text {carpet }, A} \cdot Z_{G}+\varphi_{\text {carpet }, \text { pure }} \cdot Z_{\text {carpet }, \text { pure }}+\sum_{s=1}^{3} \varphi_{p, s, \text { carpet }} \\
\cdot Z_{p, s} \\
\text { where } Z_{\text {carpet }, \text { pure }}=K_{\text {carpet }, A} \cdot Z_{G} \\
\text { and } K_{\text {carpet }, A}=K_{O A} \cdot F O C_{\text {carpet }}\end{array}$ & $\begin{array}{r}\text { (S19) } \\
\text { (S20) } \\
\text { (S21) }\end{array}$ & $\begin{array}{l}\text { - } \varphi_{\text {carpet }, A}: \text { volume fraction of } \\
\text { pore air in bulk carpet } \\
\text { - } \varphi_{\text {carpet,pure }}: \text { volume fraction } \\
\text { of pure carpet material in bulk } \\
\text { carpet } \\
\text { - } Z_{\text {carpet,pure }}: \text { fugacity capacity } \\
{\left[\mathrm{mol}^{-3} \cdot \mathrm{m}^{-3} \cdot \mathrm{Pa}^{-1} \text { of pure carpet }\right.} \\
\text { - } K_{\text {carpet }, A}: \text { carpet-air partition } \\
\text { coefficient [unitless] } \\
\text { FOC } C_{\text {carpet }}: \text { fraction of organic } \\
\text { carbon in carpet }\end{array}$ \\
\hline
\end{tabular}

(Continued on next page) 
Table S6 Continued

\begin{tabular}{|c|c|c|c|}
\hline Phase & Equation & & Parameter Description \\
\hline $\begin{array}{l}\text { Surface film }(j=\text { up-facing or } \\
\text { other surfaces) }\end{array}$ & $\begin{array}{c}Z_{f i l m, j}=\varphi_{\text {film }, \text { pure }, j} \cdot Z_{\text {film }, \text { pure }}+\sum_{s=1}^{3} \varphi_{p, s, \text { film.j }} \cdot Z_{p, s} \\
\text { where } Z_{f i l m, \text { pure }}=K_{\text {film }, A} \cdot Z_{G} \\
\text { and } K_{\text {film }, A}=0.48 \cdot K_{O A} \cdot F O C_{f i l m} \cdot \rho_{f i l m}\end{array}$ & $\begin{array}{l}(\mathrm{S} 22) \\
(\mathrm{S} 23) \\
(\mathrm{S} 24)\end{array}$ & $\begin{array}{l}\text { - } \varphi_{\text {film,pure, }, j}: \text { volume fraction of } \\
\text { pure surface film in bulk } \\
\text { surface } j \\
\text { - } Z_{\text {film,pure }}: \text { fugacity capacity } \\
{\left[\mathrm{mol} \cdot \mathrm{m}^{-3} \cdot \mathrm{Pa}^{-1}\right] \text { of pure surface }} \\
\text { film } \\
\text { - } K_{\text {film, }, A}: \text { film-air partition } \\
\text { coefficient [unitless }]^{16} \\
\text { - } F O C_{\text {film }}: \text { fraction of organic } \\
\text { carbon in surface film } \\
\text { - } \rho_{\text {film }}: \text { density of surface film } \\
{\left[\mathrm{kg} \cdot \mathrm{L}^{-1}\right]}\end{array}$ \\
\hline $\begin{array}{l}\text { Lipids }(j=\text { hands, rest of skin, } \\
\text { or internal body) }\end{array}$ & $Z_{\text {lipid }, j}=Z_{W} \cdot K_{O W}$ & (S25) & $\begin{array}{l}K_{O W}: \text { Unitless equilibrium air- } \\
\text { water partition coefficient for } \\
\text { compartment } j \text { at assumed } \\
\text { temperature }\end{array}$ \\
\hline Internal body & $Z_{\text {body }}=Z_{\text {lipid,body }} \cdot \varphi_{\text {lipid,body }}+Z_{N L O M} \cdot \varphi_{N L O M, b o d y}+Z_{W} \cdot \varphi_{W, \text { body }}$ & (S26) & $\begin{array}{l}\varphi_{\text {lipid,body }}, \varphi_{N L O M, b o d y}, \\
\varphi_{W, \text { body }}: \text { volume fractions of } \\
\text { lipids, non-lipid organic matter, } \\
\text { and water in bulk internal } \\
\text { body, respectively }\end{array}$ \\
\hline
\end{tabular}

Note: Equations were adopted from Mackay and previous studies., ${ }^{3,17,18}$ Tables S1-S4 summarize the parameterization. 
Table S7. Equations for deriving mass-transfer coefficients [MTC, $\mathrm{m} \cdot \mathrm{h}^{-1}$ ].

\begin{tabular}{|c|c|c|c|}
\hline Phase & Equation & & Parameter Description \\
\hline Air & $\begin{aligned} M T C_{A} & =\frac{B_{A}}{\delta_{b l}} \\
\text { where } B_{A} & =\frac{0.588}{M W^{0.65}}\end{aligned}$ & $\begin{array}{l}(\mathrm{S} 27) \\
(\mathrm{S} 28)\end{array}$ & $\begin{array}{l}\text { - } B_{A}: \text { Molecular diffusivity in air } \\
{\left[\mathrm{m}^{2} \cdot \mathrm{h}^{-1}\right] \text { based on an empirical }} \\
\text { equation by Schwarzenbach et } \\
\text { al. }(2003)^{19} \\
\text { - } \quad M W: \text { molecular weight } \\
{\left[\mathrm{g} \cdot \mathrm{mol}^{-1}\right]} \\
\delta_{b l}: \text { thickness of the air-side } \\
\text { boundary layer }\end{array}$ \\
\hline PUF & $\begin{array}{c}M T C_{P U F}=\frac{B_{E, P U F}}{\delta_{P U F}} \\
\text { where } B_{E, P U F}=\frac{1}{1+r_{P U F, A} \cdot K_{P U F, A}} \cdot c\end{array}$ & $\begin{array}{l}(\mathrm{S} 29) \\
(\mathrm{S} 30)\end{array}$ & $\begin{array}{l}\text { - } \\
B_{E, P U F}: \text { effective diffusivity in } \\
\text { PUF }\left[\mathrm{m}^{2} \cdot \mathrm{h}^{-1}\right] \\
\delta_{P U F}: \text { average path length for } \\
\text { diffusion in PUF }[\mathrm{m}] \\
\text { calculated as } h_{P U F} \cdot \ln (2) \\
\text { where } h_{P U F} \text { is the thickness of } \\
\text { PUF } \\
\text { - } \quad r_{P U F, A}: \text { volume ratio between } \\
\text { solid PUF material and porous } \\
\text { air in PUF } \\
\quad c: \text { correction factor accounting } \\
\text { for intra-aggregate porosity and } \\
\text { tortuosity } \\
\text { Central estimates from Zhang } \\
\text { et al. }(2011)^{4} \text { were used for } \\
r_{P U F, A} \text { and } c\end{array}$ \\
\hline
\end{tabular}

(Continued on next page) 
Table S7 Continued

\begin{tabular}{|c|c|c|c|}
\hline Phase & Equation & & Parameter Description \\
\hline Hard floor & $\begin{array}{c}M \operatorname{MT}_{\text {floor }}=\frac{B_{\text {floor }}}{\delta_{\text {floor }}} \\
\text { where } B_{\text {floor }}=\exp \left(A_{\text {pol }}-0.1351 \cdot M W^{\frac{2}{3}}+0.003 M W-\frac{10454}{T}\right) \cdot 3600\end{array}$ & $\begin{array}{l}\text { (S31) } \\
\text { (S32) }\end{array}$ & $\begin{array}{ll}- & B_{\text {floor }}: \text { Molecular diffusivity in } \\
& \text { hard floor }\left[\mathrm{m}^{2} \cdot \mathrm{h}^{-1}\right] \\
\text { - } & \delta_{\text {floor }}: \text { average path length for } \\
& \text { diffusion in hard floor [m] } \\
\text { calculated in a similar manner } \\
\text { as } \delta_{P U F} \text { above } \\
\text { - } \quad \text { Equation } S 32 \text { and } \\
\text { parameterization from Begley } \\
\text { et al. }(2005)^{20} \text { for semi- } \\
\text { crystalline polymers, e.g., high- } \\
\text { density polyethylene and } \\
\text { polyethylene terephthalate } \\
\text { - } A_{\text {pol }}: \text { a value of } 1577 \text { was used } \\
\text { - } \quad 3600: \text { conversion factor from } \\
\text { seconds to hours }\end{array}$ \\
\hline Carpet & $\begin{array}{c}M T C_{\text {carpet }}=\frac{B_{E, \text { carpet }}}{\delta_{\text {carpet }}} \\
\text { where } B_{E, \text { carpet }}=\frac{1}{1+r_{\text {carpet }, A \cdot K_{\text {carpet }, A}}} .\end{array}$ & $\begin{array}{l}\text { (S33) } \\
\text { (S34) }\end{array}$ & $\begin{array}{l}\text { - As for PUF with the only } \\
\text { difference being in the value } \\
\text { for } K_{\text {carpet }, A} \text { versus } K_{P U F, A} \\
\delta_{\text {carpet }} \text { : average path length for } \\
\text { diffusion in carpet [m] } \\
\text { calculated in a similar manner } \\
\text { as } \delta_{\text {floor }} \text { and } \delta_{P U F} \text { above }\end{array}$ \\
\hline Surface films & $\log _{10}\left(M T C_{\text {film }}\right)=0.704 \cdot \log _{10}\left(K_{O W}\right)-11.2-\log _{10}\left(K_{A W}\right)$ & (S35) & $\begin{array}{l}\text { Empirical equation adopted } \\
\text { from Diamond et al. }(2001)^{21}\end{array}$ \\
\hline
\end{tabular}

(Continued on next page) 
Table S7 Continued

\begin{tabular}{|c|c|c|c|}
\hline Phase & Equation & & Parameter Description \\
\hline $\begin{array}{l}\text { Hands \& rest of skin (dermal } \\
\text { uptake) }\end{array}$ & $\begin{array}{c}M T C_{p_{-} l}=\frac{1}{K_{L W}} \cdot M T C_{p w} \cdot 36 \\
\text { where } M T C_{p w}=\frac{M T C_{p_{-} w}}{1+B} \\
\text { where } \log _{10}\left(M T C_{p_{-} c w}\right)=0.7 \cdot \log _{10}\left(K_{O W}\right)-0.0722 \cdot M W^{2 / 3} \\
\text { and } B=\frac{M T C_{p_{-} c w} \cdot M W^{1 / 2}}{2.6}\end{array}$ & $\begin{array}{l}\text { (S36) } \\
(\mathrm{S} 37) \\
(\mathrm{S} 38) \\
(\mathrm{S} 39)\end{array}$ & 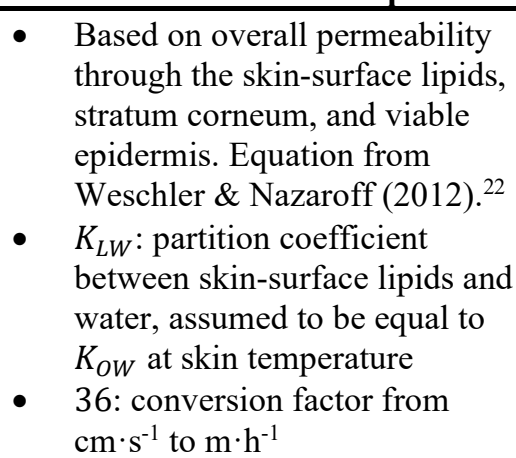 \\
\hline
\end{tabular}

Note: Equations were adopted from Mackay and previous studies. ${ }^{3,17,18}$ Tables S1, S2, and S4 summarize the parameterization. 
Table S8. Equations for deriving $D$ values $\left[\mathrm{mol} \cdot \mathrm{Pa}^{-1} \cdot \mathrm{h}^{-1}\right]$.

\begin{tabular}{|c|c|c|c|}
\hline Process & Equation & & Parameter Description \\
\hline $\begin{array}{l}\text { Diffusion between air and } \\
\text { environmental compartment } j \\
\text { where } j=\text { PUF, hard floor, carpet, } \\
\text { up-facing surfaces, or other } \\
\text { surfaces }\end{array}$ & $D_{V, A \leftrightarrow j}=\frac{1}{\frac{1}{A_{j} \cdot M T C_{A} \cdot Z_{G}}+\frac{1}{A_{j} \cdot M T C_{j} \cdot Z_{j}}}$ & $(\mathrm{~S} 40)$ & $\begin{array}{l}\text { - } A_{j} \text { : planar surface area of } \\
\text { compartment } j\left[\mathrm{~m}^{2}\right] \\
Z_{j} \text { : fugacity capacity of } \\
\text { compartment } j\left[\mathrm{~mol} \cdot \mathrm{m}^{-3} \cdot \mathrm{Pa}^{-1}\right] \text {, } \\
\text { which is } Z_{G} \text { for PUF and carpet } \\
\text { and } Z_{j, p u r e} \text { for the remaining } \\
\text { compartments }\end{array}$ \\
\hline $\begin{array}{l}\text { Diffusion from air or PUF to skin- } \\
\text { surface lipids in compartment } j \text {, } \\
\text { where } j=\text { hands or the rest of skin }\end{array}$ & $D_{V, A \leftrightarrow \text { lipid }, j}=A_{j} \cdot M T C_{A} \cdot Z_{G}$ & (S41) & $\begin{array}{l}\text { The same equation was used to } \\
\text { quantify diffusion of } \\
\text { compounds from PUF to skin- } \\
\text { surface lipids during sleeping, } \\
\text { except } M T C_{P U F} \text { was used } \\
\text { instead of } M T C_{A}\end{array}$ \\
\hline $\begin{array}{l}\text { Diffusion from skin-surface lipids } \\
\text { to the internal body from } \\
\text { compartment } j \text {, where } j=\text { hands or } \\
\text { the rest of skin }\end{array}$ & $D_{V, l i p i d, j \leftrightarrow \text { body }}=A_{\text {lipid }, j} \cdot M T C_{p_{-} L} \cdot Z_{\text {lipid }}$ & $(\mathrm{S} 42)$ & $\begin{array}{l}\text { - } \quad A_{\text {lipid }, j}: \text { surface area of skin- } \\
\text { surface lipids }\end{array}$ \\
\hline $\begin{array}{l}\text { Particle advection from } \\
\text { compartment } i \text { to compartment } j \text { for } \\
\text { particle size range } s\end{array}$ & $D_{p, s, i \rightarrow j}=V_{i} \cdot \sum_{s=1}^{3} Z_{p, s} \cdot \varphi_{p, s, i} \cdot k_{i \rightarrow j}$ & (S43) & $\begin{array}{l}k_{i \rightarrow j}: \text { first-order rate coefficient } \\
{\left[\mathrm{h}^{-1}\right] \text { for particle mass transfer }} \\
\text { (resuspension or deposition) or } \\
\text { removal }(i=j)\end{array}$ \\
\hline $\begin{array}{l}\text { Air exchange from compression } \\
\text { and release of PUF during bouncing }\end{array}$ & $D_{\text {bounce }}=V_{P U F} \cdot \varphi_{P U F, A} \cdot \varphi_{P U F, A, \text { bounce }} \cdot n_{\text {bounce }} \cdot Z_{G}$ & (S44) & $\begin{array}{l}\text { - } V_{P U F}: \text { volume of bulk PUF }\left[\mathrm{m}^{3}\right] \\
\varphi_{P U F, A, \text { bounce }}: \text { fraction of bulk } \\
\text { PUF volume that is } \\
\text { compressed during one bounce } \\
{\left[\mathrm{m}^{3} \cdot \mathrm{m}^{-3}\right]} \\
n_{\text {bounce }}: \text { frequency of bouncing } \\
{\left[\mathrm{h}^{-1}\right]}\end{array}$ \\
\hline
\end{tabular}

(Continued on next page) 
Table S8 Continued

\begin{tabular}{|c|c|c|c|}
\hline Process & Equation & & Parameter Description \\
\hline $\begin{array}{l}\text { Inhalation of gas and particles (air } \\
\text { to body) }\end{array}$ & $D_{A \rightarrow \text { body }}=G_{\text {resp }} \cdot\left(Z_{G}+\sum_{s=1}^{3} \varphi_{p, s, A} \cdot Z_{p, s}\right)$ & (S45) & - $\quad G_{\text {resp }}:$ respiration rate $\left[\mathrm{m}^{3} \cdot \mathrm{h}^{-1}\right]$ \\
\hline $\begin{array}{l}\text { Nondietary ingestion via hand-to- } \\
\text { mouth contact }\end{array}$ & $D_{\text {lipid } \rightarrow \text { body }}=n_{\text {contact }} \cdot A_{\text {contact }} \cdot h_{S S L} \cdot T E \cdot Z_{\text {lipid }}$ & (S46) & $\begin{array}{l}\text { - } n_{\text {contact }}: \text { frequency of hand- } \\
\text { mouth contact events during a } \\
\text { given interval }\left[\mathrm{h}^{-1}\right] \\
\text { - } A_{\text {contact }}: \text { area of hands } \\
\text { available for contact }\left[\mathrm{m}^{2}\right] . \\
\text { - } \quad h_{S S L}: \text { thickness of skin-surface } \\
\text { lipids [m] } \\
\text { - } \quad T E: \text { transfer efficiency, i.e., } \\
\text { fraction of chemical mass } \\
\text { transferred per contact event } \\
\text { - Equation adopted from Cohen } \\
\text { Hubal et al. }(2000)^{23} \\
\end{array}$ \\
\hline $\begin{array}{l}\text { Degradation/reaction in } \\
\text { compartment } i \text { (indoor air, PUF, } \\
\text { hard floor, carpet, up-facing } \\
\text { surfaces, other surfaces) }\end{array}$ & $\begin{array}{c}D_{\text {deg }, i}=k_{\text {deg }, i} \cdot V_{i} \cdot Z_{i, p u r e} \\
\text { where } k_{\text {deg }, i}=\frac{\log (2)}{\tau_{\text {deg }, i}}\end{array}$ & $\begin{array}{l}(\mathrm{S} 47) \\
(\mathrm{S} 48)\end{array}$ & $\begin{array}{ll}- & k_{\text {deg, }, i}: \text { pseudo first-order } \\
& \text { reaction rate coefficient }\left[\mathrm{h}^{-1}\right] \\
\text { - } & \tau_{\text {deg, }, i}: \text { degradation/reaction } \\
& \text { half-life }[\mathrm{h}]\end{array}$ \\
\hline $\begin{array}{l}\text { Degradation/reaction in the human } \\
\text { body (metabolism) }\end{array}$ & $\begin{array}{c}D_{d e g, b o d y}=k_{d e g, b o d y} \cdot V_{b o d y} \cdot Z_{\text {body }} \\
\text { where } k_{d e g, b o d y}=\frac{\log (2)}{\tau_{d e g, b o d y}}\end{array}$ & (S49) & $\begin{array}{l}\text { - } k_{\text {deg,body }}: \text { pseudo first-order } \\
\text { reaction rate coefficient }\left[\mathrm{h}^{-1}\right] \\
\text { - } V_{\text {body }}: \text { bulk volume }\left[\mathrm{m}^{3}\right] \text { of the } \\
\text { human occupant } \\
\tau_{\text {deg,body }}: \text { human metabolic } \\
\text { half-life }[\mathrm{h}]\end{array}$ \\
\hline
\end{tabular}

(Continued on next page) 
Table S8 Continued

\begin{tabular}{|c|c|c|c|}
\hline Process & Equation & & Parameter Description \\
\hline $\begin{array}{l}\text { Removal from body via exhalation } \\
\text { (gas only) }\end{array}$ & $D_{\text {rem,exhale }}=G_{\text {resp }} \cdot Z_{G}$ & $(\mathrm{~S} 51)$ & \\
\hline $\begin{array}{l}\text { Removal from body via urinary } \\
\text { excretion }\end{array}$ & $D_{\text {rem,urine }}=G_{\text {urine }} \cdot Z_{W}$ & $(\mathrm{~S} 52)$ & $\begin{array}{l}\text { - } G_{\text {urine }}: \text { urinary excretion rate } \\
{\left[\mathrm{m}^{3} \cdot \mathrm{h}^{-1}\right]}\end{array}$ \\
\hline $\begin{array}{l}\text { Removal from skin-surface lipids } \\
\text { via skin-cell regeneration }\end{array}$ & $D_{\text {rem }, c}=k_{\text {rem }, \text { skin }} \cdot V_{\text {lipid }, j} \cdot Z_{\text {lipid }}$ & (S53) & $\begin{array}{l}\text { - } k_{\text {rem,skin }}: \text { removal rate } \\
\text { coefficient }\left[\mathrm{h}^{-1}\right] \text { for skin-cell } \\
\text { regeneration }\end{array}$ \\
\hline $\begin{array}{l}\text { Removal from skin-surface lipids } \\
\text { via handwashing or showering }\end{array}$ & $D_{\text {rem,wash }}=k_{\text {rem,wash }} \cdot V_{\text {lipid }, j} \cdot Z_{\text {lipid }}$ & (S54) & $\begin{array}{l}\text { - } k_{\text {rem,wash }}: \text { removal rate } \\
\text { coefficient }\left[\mathrm{h}^{-1}\right] \text { for washing } \\
\text { skin }\end{array}$ \\
\hline
\end{tabular}

Note: Equations were adopted from Mackay and previous studies. ${ }^{3,17,18}$ Tables S1-S4 summarize the parameterization. Tables S5-S7 contain additional parameter descriptions. 


\section{Supplemental Figures}
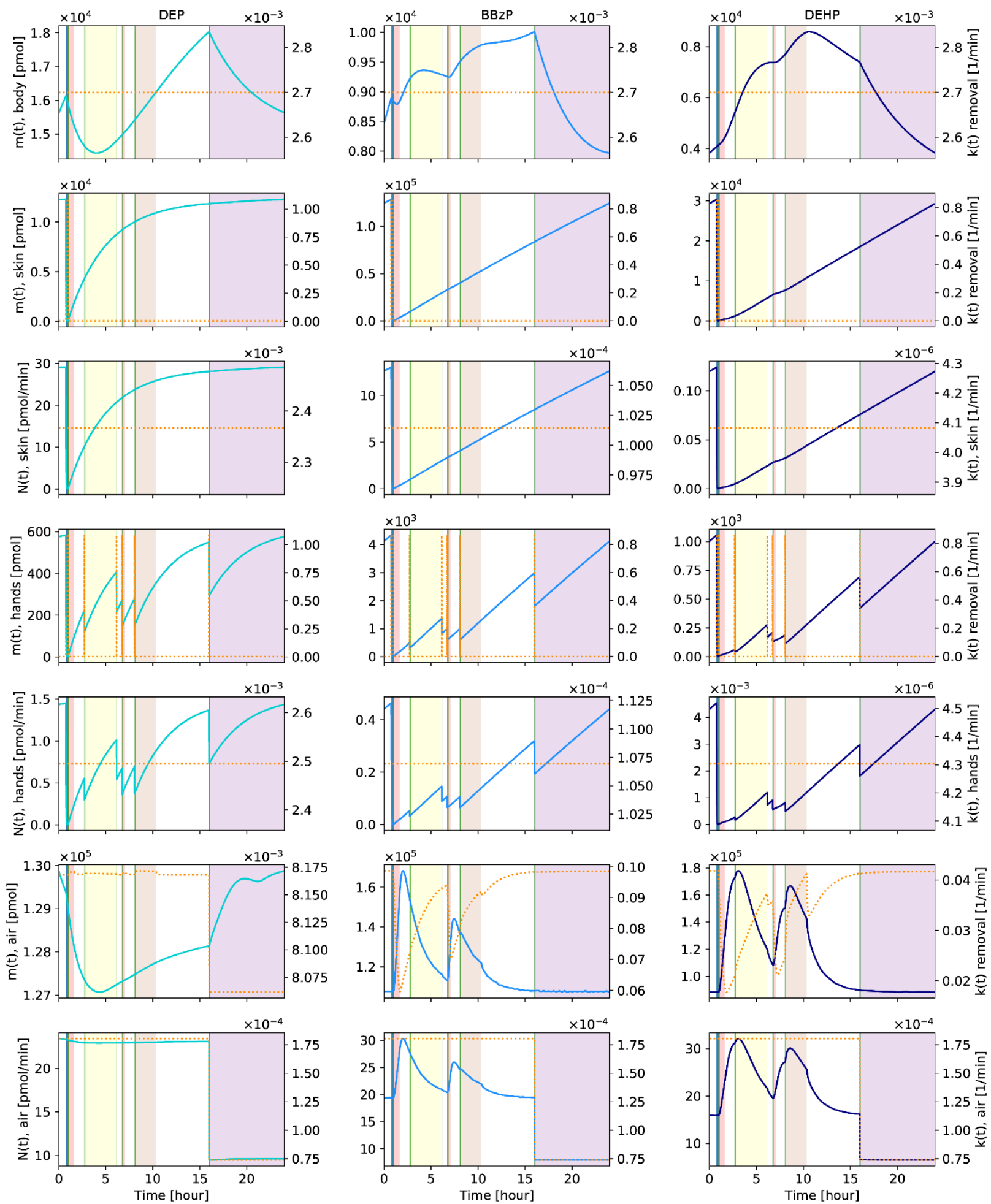

Figure S1. Dynamics of internal and external phthalate exposure for the archetypal adult male occupant. Solid (blue) lines represent the amount of phthalate $[\mathrm{m}(\mathrm{t}), \mathrm{pmol}]$ in the specified compartment, or the flux $[\mathrm{N}(\mathrm{t})$, $\left.\mathrm{pmol} \cdot \mathrm{min}^{-1}\right]$ to the internal body. Dashed (red) lines represent the corresponding removal or transfer rate coefficients 
$\left[\mathrm{k}(\mathrm{t})\right.$, pmol transferred or removed per minute per pmol in the source compartment, simplified as $\left.\mathrm{min}^{-1}\right]$. Timedependent activities (Table 1) are indicated by colored rectangles.
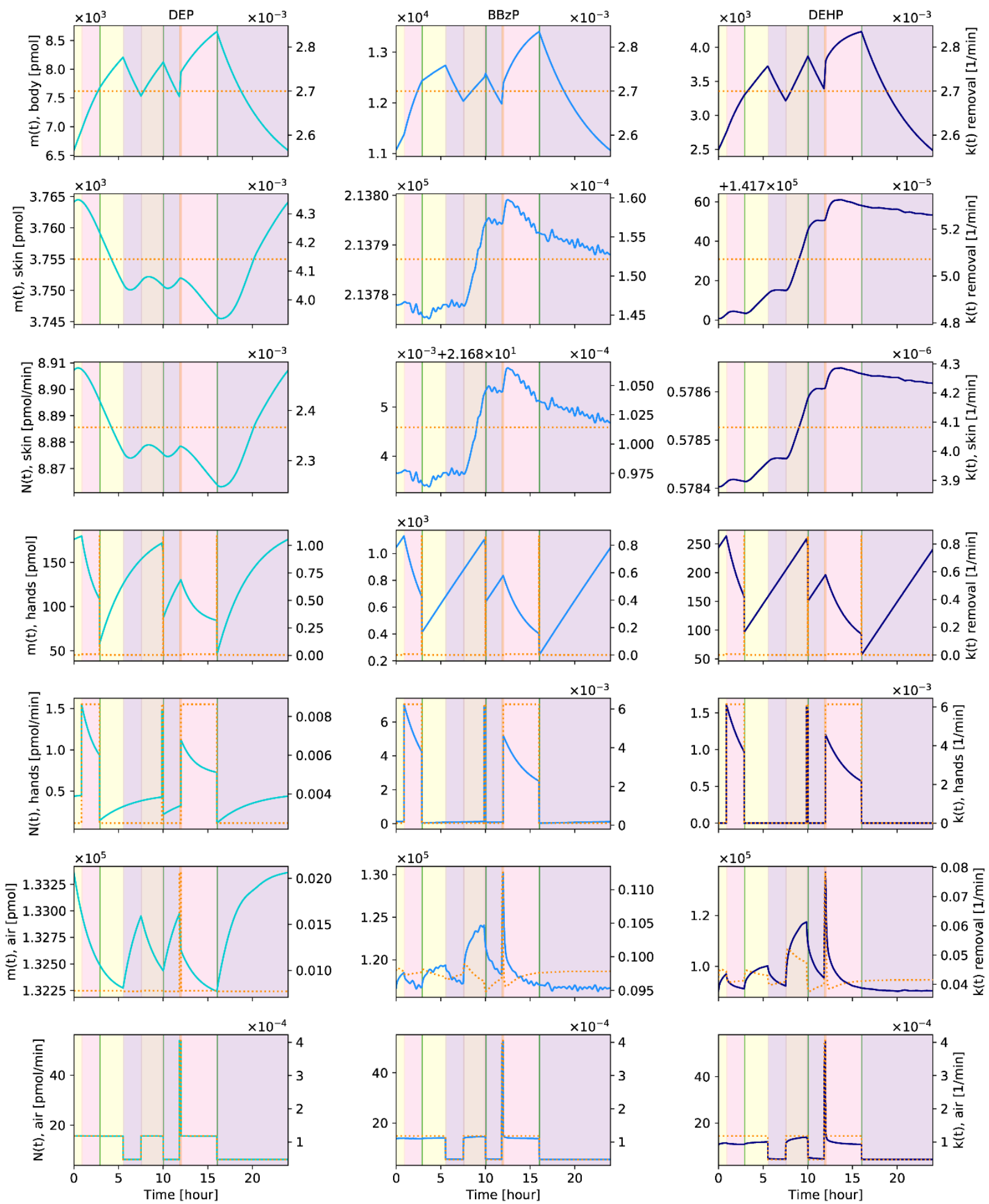

Figure S2. Dynamics of internal and external phthalate exposure for the archetypal toddler occupant. Solid (blue) lines represent the amount of phthalate $[\mathrm{m}(\mathrm{t}), \mathrm{pmol}]$ in the specified compartment, or the flux $\left[\mathrm{N}(\mathrm{t}), \mathrm{pmol} \cdot \mathrm{min}^{-1}\right]$ to 
the internal body. Dashed (red) lines represent the corresponding removal or transfer rate coefficients $[\mathrm{k}(\mathrm{t})$, pmol transferred or removed per minute per pmol in the source compartment, simplified as $\left.\mathrm{min}^{-1}\right]$. Time-dependent activities (Table 1) are indicated by colored rectangles.

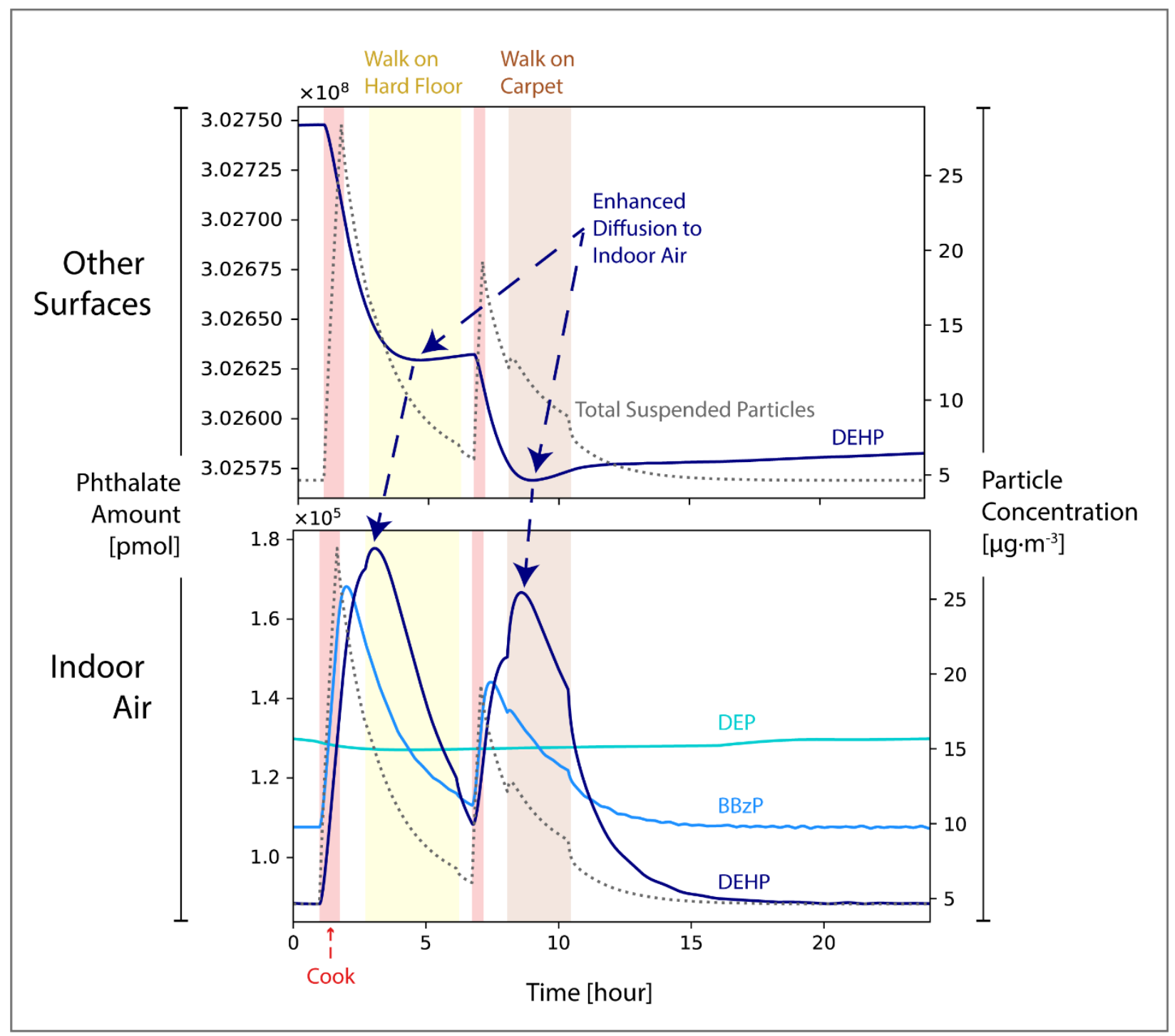

Figure S3. Dynamics of phthalates in other surfaces (top graph) and indoor air (bottom graph) for the archetypal male adult occupant's activity exposure simulation. 


\section{References}

(1) Mackay, D. Multimedia Environmental Models: The Fugacity Approach; CRC press, 2001.

(2) Bates, M. L.; Bigot, M.; Cropp, R. A.; Engwirda, D.; Friedman, C. L.; Hawker, D. W. On the Formulation of Environmental Fugacity Models and Their Numerical Solutions. Environmental toxicology and chemistry 2016, 35 (9), 2182-2191.

(3) Zhang, X.; Arnot, J. A.; Wania, F. Model for Screening-Level Assessment of near-Field Human Exposure to Neutral Organic Chemicals Released Indoors. Environmental science \& technology 2014, 48 (20), 12312-12319.

(4) Zhang, X.; Tsurukawa, M.; Nakano, T.; Lei, Y. D.; Wania, F. Sampling Medium Side Resistance to Uptake of Semivolatile Organic Compounds in Passive Air Samplers. Environ. Sci. Technol. 2011, 45 (24), 10509-10515. https://doi.org/10.1021/es2032373.

(5) U.S. Environmental Protection Agency (EPA). Exposure Factors Handbook: 2011 Edition (EPA/600/R-09/052F); National Center for Environmental Assessment: Washington, DC, 2011.

(6) Domínguez-Romero, E.; Scheringer, M. A Review of Phthalate Pharmacokinetics in Human and Rat: What Factors Drive Phthalate Distribution and Partitioning? Drug metabolism reviews 2019, 51 (3), 314-329.

(7) Kessler, W.; Numtip, W.; Völkel, W.; Seckin, E.; Csanády, G. A.; Pütz, C.; Klein, D.; Fromme, H.; Filser, J. G. Kinetics of Di (2-Ethylhexyl) Phthalate (DEHP) and Mono (2Ethylhexyl) Phthalate in Blood and of DEHP Metabolites in Urine of Male Volunteers after Single Ingestion of Ring-Deuterated DEHP. Toxicology and applied pharmacology 2012, 264 (2), 284-291.

(8) Gong, M.; Zhang, Y.; Weschler, C. J. Measurement of Phthalates in Skin Wipes: Estimating Exposure from Dermal Absorption. Environmental science \& technology 2014, 48 (13), 7428-7435.

(9) Shin, H.-M.; McKone, T. E.; Tulve, N. S.; Clifton, M. S.; Bennett, D. H. Indoor Residence Times of Semivolatile Organic Compounds: Model Estimation and Field Evaluation. Environmental science \& technology 2013, 47 (2), 859-867.

(10) Bramwell, L.; Qian, J.; Howard-Reed, C.; Mondal, S.; Ferro, A. R. An Evaluation of the Impact of Flooring Types on Exposures to Fine and Coarse Particles within the Residential Micro-Environment Using CONTAM. Journal of Exposure Science and Environmental Epidemiology 2016, 26 (1), 86.

(11) Boor, B. E.; Spilak, M. P.; Corsi, R. L.; Novoselac, A. Characterizing Particle Resuspension from Mattresses: Chamber Study. Indoor Air 2015, 25 (4), 441-456.

(12) Qian, J.; Ferro, A. R. Resuspension of Dust Particles in a Chamber and Associated Environmental Factors. Aerosol Science and Technology 2008, 42 (7), 566-578.

(13) Popkin, B. M.; D’Anci, K. E.; Rosenberg, I. H. Water, Hydration, and Health. Nutrition reviews 2010, 68 (8), 439-458.

(14) U.S. EPA. Standard Operating Procedures for Residential Pesticide Exposure Assessment; US Environmental Protection Agency, Office of Pesticide Programs Washington, DC, 2012.

(15) Harner, T.; Bidleman, T. F. Octanol- Air Partition Coefficient for Describing Particle/Gas Partitioning of Aromatic Compounds in Urban Air. Environmental Science \& Technology 1998, 32 (10), 1494-1502. 
(16) Diamond, M. L.; Gingrich, S. E.; Fertuck, K.; McCarry, B. E.; Stern, G. A.; Billeck, B.; Grift, B.; Brooker, D.; Yager, T. D. Evidence for Organic Film on an Impervious Urban Surface: Characterization and Potential Teratogenic Effects. Environ. Sci. Technol. 2000, 34 (14), 2900-2908.

(17) Zhang, X.; Diamond, M. L.; Ibarra, C.; Harrad, S. Multimedia Modeling of Polybrominated Diphenyl Ether Emissions and Fate Indoors. Environ. Sci. Technol. 2009, 43 (8), 2845-2850. https://doi.org/10.1021/es802172a.

(18) Li, L.; Westgate, J. N.; Hughes, L.; Zhang, X.; Givehchi, B.; Toose, L.; Armitage, J. M.; Wania, F.; Egeghy, P.; Arnot, J. A. A Model for Risk-Based Screening and Prioritization of Human Exposure to Chemicals from near-Field Sources. Environmental science \& technology 2018.

(19) Schwarzenbach, R. P.; Gschwend, P. M.; Imboden, D. M. Environmental Organic Chemistry: Second Edition; John Wiley \& Sons, 2003.

(20) Begley, T.; Castle, L.; Feigenbaum, A.; Franz, R.; Hinrichs, K.; Lickly, T.; Mercea, P.; Milana, M.; O’Brien, A.; Rebre, S. Evaluation of Migration Models That Might Be Used in Support of Regulations for Food-Contact Plastics. Food Additives and Contaminants 2005, 22 (1), 73-90.

(21) Diamond, M. L.; Priemer, D. A.; Law, N. L. Developing a Multimedia Model of Chemical Dynamics in an Urban Area. Chemosphere 2001, 44 (7), 1655-1667.

(22) Weschler, C. J.; Nazaroff, W. W. SVOC Exposure Indoors: Fresh Look at Dermal Pathways. Indoor Air 2012, 22 (5), 356-377.

(23) Cohen Hubal, E. A.; Sheldon, L. S.; Burke, J. M.; McCurdy, T. R.; Berry, M. R.; Rigas, M. L.; Zartarian, V. G.; Freeman, N. C. Children's Exposure Assessment: A Review of Factors Influencing Children's Exposure, and the Data Available to Characterize and Assess That Exposure. Environmental health perspectives 2000, 108 (6), 475-486. 